Abstract 178 Table 1 Association of laboratory and clinical values (SLAM) with SLE flares

\begin{tabular}{lllll}
\hline & Correlation coefficient & $\mathbf{p}$ & RR & $95 \% \mathrm{Cl}$ \\
\hline ESR & 0.34 & $<0.05$ & 1.56 & $0.36-0.87$ \\
ANA & 0.22 & $>0.05$ & 1.12 & $0.43-1.50$ \\
AntiDNA Ab & 0.13 & $>0.05$ & 0.98 & $0.67-2,13$ \\
Low Hb level & 0.48 & $<0.05$ & 1.99 & $0.45-0.80$ \\
Low leucocytes & 0.23 & $>0.05$ & 1.01 & $0.56-1.33$ \\
Low lymphocytes & 0.56 & $<0.05$ & 2.05 & $0.33-0.67$ \\
Antiphospholipid syndrome & - & - & 2.30 & $0.61-0.88$ \\
Pulmonary Involvement & - & - & 1.88 & $0.23-0.82$ \\
\hline
\end{tabular}

During a 12 moths fallow-up, 55 flares were enregistered, including 11 cases of severe flares, with a SLEDAI increase from 3 to 17 points. So, the total incidence of flares was 0,53 patient/year and the incidence for severe flares was 0,10 patient/year. In order to assess the risk of flares, we have studied several potential risk factors, as shown in the table.

Conclusions the incidence of flare in a 12 months period was $53,9 \%$, including $10,8 \%$ of severe flares. Low $\mathrm{Hb}$ and lymphocytopenia are at risk for flares and antiphospholipid syndrome and pulmonary involvement were the main clinical risk factors in our cohort.

\section{BIOMARKERS OF ATHEROSCLEROSIS IN SLE IMPROVE AFTER TREATMENT WITH MYCOPHENOLATE MOFETIL}

M McMahon*, B Skaggs, J Grossman, L Sahakian, B Hahn. UCLA David Geffen School of Medicine, Rheumatology, Los Angeles, USA

\subsection{6/lupus-2017-000215.179}

Background and aims Women with SLE have an increased risk of atherosclerosis that is not adequately explained by traditional risk factors. We previously discovered that a "high risk" score on a panel of biomarkers, PREDICTS, confers 28-fold increased odds for carotid plaque in SLE women, and is also associated with IMT progression. The biomarkers included are pro-inflammatory HDL, sTWEAK $\geq 373 \mathrm{pg} / \mathrm{mL}$, homocysteine $\geq 12 \mathrm{mmol} / \mathrm{L}$, leptin $\geq 34 \mathrm{ng} / \mathrm{dL}$, age $\geq 48$ years, and DMII. It is unknown, however, whether these biomarkers are modifiable by SLE disease modifying agents.

Methods This prospective observational study included UCLA cohort patients started on new immunosuppressive agents. Plasma samples were taken at baseline and 12 weeks. HDL antioxidant function was measured by changes in fluorescence intensity of a substrate incubated with LDL and patient HDL. Plasma leptin and sTWEAK were measured using ELISA. Homocysteine was measured in the UCLA clinical labs.

Results 16 subjects started mycophenolate mofetil (MMF), 18 azathioprine (AZA), and 25 hydroxychloroquine (HCQ). In MMF treated subjects, HDL function ( $p=0.009$, paired $t$-test) and sTWEAK $(p=0.05)$ significantly improved after 12 weeks, while leptin and homocysteine did not significantly change. In HCQ treated subjects, HDL function improved $(p=0.05)$. In the AZA group there were no significant changes in any of the biomarkers. Overall, the mean number of PREDICTS biomarkers at week 12 significantly decreased in the MMF group $(\mathrm{p}=0.03)$.
Conclusions The mean number of "high-risk" cardiac biomarkers significantly improved after initiation of MMF. Further longitudinal studies will determine whether changes in biomarkers reflect decreased cardiovascular events.

\section{PLASMA MYELOPEROXIDASE IS INVERSELY ASSOCIATED WITH FUTURE ATHEROSCLEROSIS PROGRESSION AND INFLAMMATORY HDL FUNCTION IN SLE}

M McMahon*, J Grossman, B Hahn, B Skaggs. UCLA David Geffen School of Medicine, Rheumatology, Los Angeles, USA

\subsection{6/lupus-2017-000215.180}

Background and aims Women with SLE have increased atherosclerosis (ATH) that is not adequately explained by traditional risk factors. We previously discovered that a "high risk" score on a panel of biomarkers, PREDICTS, confers 28-fold increased odds for carotid plaque in SLE women. The biomarkers included in PREDICTS are sTWEAK, pro-inflammatory HDL (piHDL), homocysteine, leptin, age $\geq 48$, and DMII. It is unknown, however, whether other biomarkers of oxidative stress also predict progression of ATH in SLE. The enzyme myeloperoxidase (MPO) catalyses formation of reactive oxygen species and generates piHDL. The aim of this study was to determine whether MPO levels might predict future progression of ATH in SLE.

Methods B-mode and Doppler scanning of carotid arteries was performed at baseline and 24-36 months. Baseline plasma MPO levels were measured using ELISA.

Results Repeat carotid ultrasounds and MPO measurements were completed on 202 SLE women. Plaque progression (defined as new or increased plaque) was seen in 42 subjects (21\%). Baseline MPO levels were significantly lower in SLE patients with plaque progression vs. those without $(\mathrm{p}<0.001)$. Baseline MPO levels were also inversely correlated with piHDL function at follow-up $(\mathrm{r}=-0.33, \mathrm{p}<0.001)$. Using logistic regression, the variables associated with plaque progression in SLE included high PREDICTS (OR 27.0 $\mathrm{p}<0.001$ ), MPO levels in the lowest half (OR 4.2, $\mathrm{p}=0.005$ ), and non-Caucasian ethnicity (OR 4.5, p=0.003).

Conclusions Plasma MPO levels are inversely associated with plaque progression in SLE. Lower baseline MPO levels are also associated with future formation of inflammatory piHDL, suggesting that this could be one mechanism to explain the association.

\section{EPIDEMIOLOGIC PROFILE OF ERECTILE DYSFUNCTION IN SLE: A MULTI-CENTRECENTER STUDY IN LATIN AMERICAN PATIENTS}

IJ Merayo-Chalico*, 'A Barrera-Vargas, 'S Morales-Padilla, 'R Reyna-de-la Garza, ${ }^{1} \mathrm{R}$ Vázquez-Rodríguez, ${ }^{2} \mathrm{M}$ Sotomayor, ${ }^{1} \mathrm{D}$ Gómez-Martín, ${ }^{1} \mathrm{~J}$ Alcocer-Varela, ${ }^{3} \mathrm{I}$ ColungaPedroza, ${ }^{4} \mathrm{C}$ Abud, ${ }^{4} \mathrm{M}$ Martínez-Martínez, ${ }^{5} \mid$ ${ }^{7}$ D Fajardo. "Instituto Nacional de Ciencias Médicas y Nutrición "Salvador Zubirán", Immunology and Rheumatology, Mexico City, Mexico; ${ }^{2}$ Instituto Nacional de Ciencias Médicas y Nutrición "Salvador Zubirán", Urology, Mexico City, Mexico; ${ }^{3}$ Hospital Universitario, Rheumatology, Monterrey, Mexico; ${ }^{4}$ Hospital Central, Rheumatology, San Luis Potosí, Mexico; ${ }^{5}$ nstituto Salvadoreño del Seguro Social, Rheumatology, San Salvador, El Salvador; ${ }^{6}$ Hospital Metropolitano Vivian Pellas, Rheumatology, Managua, Nicaragua; ${ }^{7}$ Instituto Mexicano del Seguro Social, Rheumatology, Guadalajara, Mexico

10.1136/lupus-2017-000215.181 
Abstract 181 Table 1

\begin{tabular}{|c|c|c|c|}
\hline Variable & $\begin{array}{l}\text { SLE without Erectile } \\
\text { Dysfunction } \\
(n=50) \\
\text { (mean } \pm \text { SEM) } \\
32.9 \pm 1.3\end{array}$ & $\begin{array}{c}\text { SLE with Erectile } \\
\text { Dysfunction } \\
\text { (n=107) } \\
\text { (mean } \pm \text { SEM) } \\
35.3 \pm 1\end{array}$ & pvalue \\
\hline $\mathrm{BMI}\left(\mathrm{m}^{2} / \mathrm{kg}\right)$ & $26.1 \pm 0.61$ & $27.1 \pm 0.50$ & 0.23 \\
\hline Time from SLE diagnosis (years) & $7.7 \pm 0.96$ & $7.7 \pm 0.68$ & 0.99 \\
\hline Smoking (in the last 5 years), $N(\%)$ & $11 / 50(22.4)$ & $30 / 107(28)$ & 0.85 \\
\hline Diabetes mellitus, $\mathrm{N}(\%)$ & $0 / 50(0)$ & $6 / 107(5.6)$ & 0.17 \\
\hline Hypertension, $\mathrm{N}(\%)$ & $14 / 50(28.6)$ & $37 / 107(34.9)$ & 0.46 \\
\hline Dyslipidemia, N (\%) & $11 / 50(22.4)$ & $29 / 107(27.1)$ & 0.69 \\
\hline APS, $N(\%)$ & $19 / 50(39.6)$ & $35 / 107(32.7)$ & 0.46 \\
\hline Lupus nephritis (biopsy), N (\%) & $20 / 50(40.8)$ & $61 / 107(57)$ & 0.084 \\
\hline $\begin{array}{l}\text { Persistent lymphopenia ( }=1000 \mathrm{cel} / \mathrm{mm}^{3} \text { at } \\
\text { least } 3 \text { times), } \mathrm{N}(\%) \\
\text { SLEDAl score (points) }\end{array}$ & $3.6 \pm 0.55$ & $\begin{array}{c}44 / 107(41.4) \\
5.1 \pm 0.59\end{array}$ & 0.11 \\
\hline SUCC score (points) & $0.80 \pm 0.17$ & $1.24 \pm 0.15$ & 0.07 \\
\hline Immunosuppressive therapy, $\mathrm{N}(\%)$ & $43 / 50(87.9)$ & $98 / 107(91.6)$ & 0.55 \\
\hline Cumulative prednisone dose (5 years) (mg) & $11,709 \pm 1959$ & $12,885 \pm 1289$ & 0.61 \\
\hline $\begin{array}{l}\text { Cumulative prednisone dose (last year) } \\
\text { (mg) }\end{array}$ & $2,278 \pm 596$ & $2,784 \pm 379$ & 0.46 \\
\hline $\begin{array}{l}\text { Exposure to any dose of steroids in the } \\
\text { previous year, } N(\%)\end{array}$ & $28 / 50(56)$ & $85 / 107(79.4)$ & 0.004 \\
\hline MMF dose (mg) & $549 \pm 132$ & $0.685 \pm 940$ & 0.40 \\
\hline $\begin{array}{l}\text { Cyclophosphamide cumulative dose, } \\
\text { lifelong }(\mathrm{gr})\end{array}$ & $12.07 \pm 6.7$ & $12.03 \pm 4.1$ & 0.99 \\
\hline Antimalarial, $\mathrm{N}(\%)$ & $31 / 50(62)$ & $66 / 107(61)$ & 1 \\
\hline $\begin{array}{l}\text { Any treatment for comorbidities (non- } \\
\text { imunosuppressive), } \mathrm{N}(\%)\end{array}$ & $38 / 50(76)$ & $86 / 107(80.4)$ & 0.53 \\
\hline
\end{tabular}

Background and aims Although SLE has a higher prevalence in women, the disease usually has a more aggressive course in men. Therefore, the aim of this study was to describe the prevalence of erectile dysfunction (ED), as well as associated demographic and clinical features in men with SLE, by means of a systematic, standardised evaluation.

Methods We performed a transversal study in six tertiary care centres in Latin America. We included male patients $\geq 16$ years who fulfilled $\geq 4$ ACR criteria for SLE, and had regular sexual activity in $\leq 6$ months. Patients with other rheumatic diseases (except for APS), chronic viral infections and late-onset SLE were excluded. All patients answered the IIEF-5 Questionnaire, which has been validated in Spanish. Other relevant demographic, clinical and serological characteristics were documented.

Results We included 157 subjects. The prevalence of ED in our study population was $68 \%$, the majority were classified as mild to moderate (17.3 \pm 0.36 points; normal score: $22-25$ 


\begin{tabular}{lc|c|c} 
Abstract 181 Table 2 & Multivariate analysis. \\
\hline Variable & OR & $95 \% \mathrm{Cl}$ & pvalue \\
Age (=35 years) & 1.016 & $1.001-1.032$ & 0.037 \\
\hline $\begin{array}{l}\text { Exposure to steroid in the } \\
\text { previous year }\end{array}$ & 0.364 & $0.175-0.759$ & 0.007 \\
\hline \begin{tabular}{l} 
Persistent lymphopenia \\
\hline
\end{tabular} & 2.42 & $1.102-5.344$ & \\
\hline
\end{tabular}

points). The mean age of patients with $\mathrm{ED}$ was $35.3 \pm 1$, while in patients without ED it was $32.9 \pm 1.3 \quad(p=0.17)$. The only significant differences were regarding corticosteroid usage in the past year; the rest of the demographic and clinical variables were similar between both groups. (Table 1). After multivariate analysis, independent risk factors for ED were: persistent lymphopenia, age and corticosteroid use in the past year (Table 2).

Conclusions Regardless of comorbidities, medication (excluding corticosteroids) and disease duration, SLE patients, who are mostly young and sexually active, have a high ED prevalence.

\section{ACUTE MYOSITIS AS A FLARE MANIFESTATION OF SYSTEMIC LUPUS ERYTHEMATOSUS}

K Mizushina*, T Ogura, A Hirata, T Katagiri, H Kameda. Toho University Ohashi Medical Centre, Rheumatolgy, Tokyo, Japan

\subsection{6/lupus-2017-000215.182}

Background and aims Myositis, especially acute myositis, is a rare manifestation of systemic lupus erythematosus (SLE). Here we report a case of acute myositis concomitant with lupus pleuritis.

Methods a case report and review of literature.

Results A 29-year-old woman with an 8 year history of SLE was admitted to our hospital because of pleuritic chest pain. Her initial diagnosis as SLE was made by malar rash, photosensitivity, oral ulcer, oligoarthritis, leukopenia and the positivity for antinuclear antibodies as well as anti-Sm. She has shown recurrent pleuritis afterwards. The Chest CT revealed bilateral pleural and pericardial effusion. Bacterial cultures and viral antibody tests were negative, and the daily dose of prednisolone was increased from $5 \mathrm{mg}$ to $20 \mathrm{mg}$. Despite the improvement in the pleuritic chest pain, she developed acute myalgia with the elevated value of serum muscle enzymes, positive signals in the muscle/fascia by the ultrasound and MRI, and myopathic changes in the electromyogram examination. After the administration of intravenous steroid pulse therapy for 3 days followed by prednisolone $40 \mathrm{mg} /$ day, all the myositic signs and symptoms subsided, which was also confirmed by the ultrasound.

Conclusions The present case suggests that acute myositis may develop as a manifestation of SLE exacerbation and the ultrasound evaluation may be useful in the diagnosis and the follow-up of myositis.

\section{EFFECT OF THE METABOLIC SYNDROME ON ORGAN DAMAGE AND MORTALITY IN CHINESE PATIENTS WITH SYSTEMIC LUPUS ERYTHEMATOSUS: A LONGITUDINAL ANALYSIS}

${ }^{1} \mathrm{CC}$ Mok, ${ }^{2} \mathrm{LY}$ ho, ${ }^{2} \mathrm{SM}$ Tse. ${ }^{1}$ Hong Kong S.A.R; ${ }^{2}$ Tuen Mun Hospital, Medicine, Hong Kong, Hong Kong S.A.R

\subsection{6/lupus-2017-000215.183}

Background and aims To study the effect of the metabolic syndrome(MetS) on organ damage and mortality in patients with SLE.

Methods Consecutive patients who fulfilled $\geq 4$ ACR criteria for SLE were assessed for the presence of the MetS in 2010. The MetS was defined by the updated joint consensus criteria, using the Asian criteria for central obesity. Longitudinal data on organ damage, vascular events and mortality were retrieved from our database. The association of the MetS with new organ damage and mortality was studied by logistic regression. Results 577 SLE patients were studied (93\% women; age41.2 \pm 13.4 years; SLE duration9.3 \pm 7.2 years). The mean followup time of the patients was $66.3 \pm 1.8$ months. $85(14.7 \%)$ patients qualified the MetS. New organ damage and vascular events developed in $128(22 \%)$ and 23 (4.0\%) patients, respectively. Thirty-nine (6.8\%) patients died. Patients with MetS, compared to those without, had significantly higher SDI accrual at their last visits $(0.70 \pm 1.0$ vs $0.26 \pm 0.6$; $\mathrm{p}<0.001)$. New vascular events $(11 \%$ vs $2.8 \% ; \mathrm{p}=0.001)$, allcause mortality $(14 \%$ vs $5.5 \% ; \mathrm{p}=0.003)$, death due to vascular complications $(7.1 \%$ vs $0.2 \% ; \mathrm{p}<0.001)$ were significantly more common in patients with MetS than those without. Logistic regression revealed that the MetS was significantly associated with new damage in the ocular, renal, cardiovascular and endocrine system, adjusted for age, sex, SLE duration and the antiphospholipid antibodies.The presence of the MetS showed a significant increase in vascular mortality after adjustment for the same covariates (OR 30.3 [3.42-268]; $p=0.002$ ). Conclusions The MetS is significantly associated with new organ damage, vascular events and mortality in patients with SLE.

\section{CHARACTERISTICS AND RISK FACTORS FOR TUBERCULOSIS INFECTION AMONG FILIPINO PATIENTS WITH SYSTEMIC LUPUS ERYTHEMATOSUS}

RM Molina, S Navarra. University of Santo Tomas, Internal Medicine- Section of Rheumatology, Metro Manila, Philippines

\subsection{6/lupus-2017-000215.184}

Background and aims To describe characteristics and risk factors for tuberculosis (TB) among Filipino systemic lupus erythematosus (SLE) patients.

Methods We analysed SLE patients diagnosed with TB at Lupus Clinics of University of Santo Tomas Hospital, Manila, Philippines. TB categories included pulmonary (PTB) only, extra-pulmonary (EPTB) single site, disseminated (DTB) defined as $>1$ organ involvement. Disease characteristics, cumulative steroid, average daily prednisone dose and immunosuppressive use over 3 months preceding TB diagnosis, and outcomes were compared among the 3 TB categories by oneway analysis of variance (ANOVA) and multivariate discriminant analysis. 